\title{
HEINE, SCHUBERT UND WOLF: "ICH STAND IN DUNKELN TRÄUMEN"**
}

Günter Eich hat eines der Stücke seiner Kurzprosasammlung Ein Tibeter in meinem Büro von 1970 mit der Überschrift "Beethoven, Wolf und Schubert" versehen. Dem verdankt sich die Formulierung unseres Titels.

\section{Zugänge zu einem Klavierlied nach Heine}

Heines Gedicht oder Lied "Ich stand in dunkeln Träumen" entstammt seinem insgesamt knapp hundert Gedichte umfassenden Zyklus Die Heimkebr von 1823/24, in dem ein hochromantisches, fragiles lyrisches Ich kein Bein auf die Erde bringt, - insbesondere was die Liebe als das Zentralthema betrifft. Zusammen mit fünf anderen Heine-Gedichten setzte Schubert es in seinem Todesjahr 1828 in Musik. Erschienen sind die sechs Klavierlieder nach Heine im Folgejahr in der vom Verleger unter dem Titel Schwanengesang zusammengefaßten Sammlung. Das 1826 erstmals veröffentlichte Gedicht war brandaktuell, als Schubert sich seiner annahm. Wolf wiederum schrieb sein entsprechendes Klavierlied im Mai 1878 als eines von sieben, die den "Liederstrauß" von 1878 bilden und auf Gedichte aus Heines Buch der Lieder, zu dem auch Die Heimkehr gehört, komponiert sind. Die ungefähr 15 inzwischen publik gewordenen Klavierlieder von Wolf nach Heine entstammen den Jahren zwischen 1876 und 1880, sind also - und es ist entscheidend, dies festzuhalten - frühe Kompositionen; nur eines folgte 1888, also erheblich später, nach: "Wo wird einst des Wandermüden" als erstes der Vier Gedichte

\footnotetext{
- Hier zum ersten Mal veröffentlichter Vortrag, den der Verfasser im Mai 1996 im Rahmen des von der Internationalen Hugo-WolfAkademie veranstalteten Symposiums "Musikalische Lyrik: Hugo Wolf und seine Dichter" an der Staatlichen Hochschule für Musik und Darstellende Kunst in Stuttgart (Bundesrepublik Deutschland) gehalten hat.
} 
nach Heine, Shakespeare und Byron. Wenn der Komponist seine Lieder für Klavier und Gesang hier als Gedichte bezeichnet, so kehrt er den Spieß um, der Literaten seit alters dazu brachte, ihre Gedichte Lieder zu nennen.

Mit "Ich stand in dunkeln Träumen" steht Schubert am Anfang, Wolf schon eher am Ende der ersten und mächtigen "Vertonungs"-Welle von Heine-Gedichten. Dazwischen liegen Mendelssohn, vor allem jedoch Schumann und Liszt, neben anderen aber auch etwa Hans von Bülow und der auf dem Heine-Feld schon quantitativ nicht zu unterschätzende Robert Franz. Die Gründe, warum die Woge abebbte, sind verzweigt. Als Anhaltspunkte seien nur genannt: Die Aktualität Heines war wohl verblaßt, seine Popularität blieb zwar bestehen, war aber restringiert. Es dürfte typisch sein, daß unter den Musikern ebenso Liszt wie Bülow, die Heine anfänglich vertonten, sich später distanziert über ihn geäußert haben. Nicht zu unterschätzen ist dabei - unter anderem - auch, daß der Antisemitismus sich zwischenzeitlich merklich verstärkt hat. Der Dichter Heine und mit ihm das Heine-Gedicht konnten im deutschen Kaiserreich nach 1871 und in der allmählich sterbenden habsburgischen Monarchie keine wirkliche Repräsentanz mehr für sich in Anspruch nehmen. Und zumal für einen Wagner-Jünger wie Wolf (wie übrigens auch für Bülow) könnte es von einem bestimmten Punkt an eine Rolle gespielt haben, daß Richard Wagners infame Wortkampagnen gegen Heine womöglich nicht ohne jene Wirkung geblieben sind, die sie haben erzeugen sollen.

Zwischen Schuberts spätem Versuch von 1828 und Wolfs frühem Versuch von 1878 liegt ein halbes Jahrhundert, das als kompositionsgeschichtliche Erfahrungsund Entwicklungszeit seine Spuren hinterlassen hat. Aber so sehr die Kompositionsgeschichte im allgemeinen und die Liedkomposition im besonderen sich zwischenzeitlich hörbar verändert hat, so wenig ist die Einheit zu übersehen, die das Klavierlied als Genre aufweist. Zwischen einem Lied von Schubert und einem von Wolf liegen aufs ganze gesehen weder kompositorisch noch geschichtlich trennende Welten. Auch im Blick auf "Ich stand in dunkeln Träumen" wäre es, so verständlich der Standpunkt sein mag, unangebracht, nur das Trennende ausfindig machen zu wollen.

Bei der Betrachtung von Klavierliedern richtet der Blick sich unter Musikern - aber nicht nur bei ihnen - in der Regel von der Musik (der Vertonung') zurück auf das Gedicht (die Verse). Selten ist es umgekehrt, daß vom Gedicht als dem zumeist Anfänglicheren her auf die Komposition geschaut wird. Seit Generationen lernt jemand ein Gedicht oft nur kennen, weil ein Klavierlied nach ihm verfaßt ist.

1 Der übliche Ausdruck "Vertonung" ist zwar durch und durch problematisch, aber schon insofern kaum verzichtbar, als im Deutschen ein Synonym fehlt, das ihn ersetzen könnte, ohne in sprachliche Umständlichkeiten zu geraten (z.B. durch das altmodisch gewordene "in Musik setzen", dem "to set to music" im Englischen entspricht). 
Gewöhnlich wird durch die Komposition hindurch auf das Gedicht zurückgegangen, statt - wie der Komponist - vom Gedicht zu der Komposition zu gelangen ${ }^{2}$. Maßstab bleibt das Klavierlied, nicht die Gedichtvorlage. Das hat zur Folge, daß dem Gedicht sozusagen der Anwalt fehlt, der sich fragt, was eigentlich vom Gedicht in der Musik überhaupt noch übriggeblieben ist, weil der Eindruck unabweisbar ist, daß eineitig die Frage im Vordergrund steht, was alles die Musik am Gedicht eingefangen hat. Nun sind die meisten Gedichte zuerst einmal nicht dazu geschrieben und dazu da, von einem Komponisten bearbeitet zu werden, sondern sie sind Kunstgebilde sui generis und eigenen Rechts. Heine mag von Vertonungen seiner Gedichte geschmeichelt gewesen sein: Die Poeten wissen die Zugewinne an Popularität durch Töne zu ihren oft - freilich nicht im Falle Heines - weniger populären lyrischen Produkte zu schätzen, können aber deshalb auch neidisch werden, da es für ein Gedicht als Gedicht ein zweifelhaftes Kompliment ist, erst durch jene Töne bekannt zu werden, die es im wesentlichen tangieren und unvermeidlich ramponieren.

Es ist eigenartig: Auf der einen Seite haben angehende Sängerinnen und Sänger, Liedbegleiter und Musiktheoretikerinnen, die die Lieder wiedergeben oder zum Gewinnen von Aufschlüssen über den Tonsatz in ihre musikalischen Bestandteile zerlegen, ein Anrecht darauf, daß ihnen die zugrundeliegenden Gedichte durch Erläuterung vertraut gemacht werden, um des konkreten Ganzen besser habhaft zu werden. Auf der anderen Seite sollte man den Exegeten der Musik die Gedichte vielleicht eher vorenthalten, um in den verbalen Interpretationen Wildwuchs zu vermeiden und sie davor zu bewahren, sich bei der Erklärung der Kompositionen - der Lieder als Musik - zu sehr an die Gedichte zu klammern und sich von ihnen leiten zu lassen. Wichtiger als die Interpretations-Absicht der Exegeten dürfte der Versuch einer Rekonstruktion dessen sein, wie der Komponist selbst die Vorlage (das Gedicht) analysiert hat; denn dies ist, wie etwa Luciano Berio betont hat, ausschlaggebend für das musikalische Ergebnis, - natürlich im Rahmen der der jeweiligen Zeit zur Verfügung stehenden bzw. gängigen kompositionstechnischen Mittel und (musik)historischen Rahmenbedingungen. Um eine Hypothese etwas zugespitzt zu formulieren: Vielleicht sollte bei der Besprechung von Klavierliedern zwischen Schubert und Mahler oder Richard Strauss weniger das ins Zentrum gerückt werden, was am Text des Gedichts "getroffen", "eingeholt", gar verdoppelt ist, als vielmehr die Frage, ob ein treffliches, in sich stimmiges Musikstück entstanden ist. Demgegenüber kann es möglicherweise marginal bleiben, wie nahe das Musikstück sich an das Gedicht hält.

2 Eine andere Perspektive ergibt sich, wenn ein Klavierlied (wie etwa die meisten Schlager) so zustande kommt, daß ein Gedicht der Musik (nachträglich) unterlegt wird, statt daß die Verse vertont werden, - ein Verfahren, von dem bei Komponisten wie Schubert und Wolf nicht auszugehen ist. 
Unter den zahlreichen Erläuterungsstrategien, die bei (traditionellen) Klavierliedern des 19. Jahrhunderts verfolgt werden können, stechen drei hervor, obwohl sie selten unvermischt begegnen. Die erste geht von der Annahme aus, daß ein solches Klavierlied etwas von den Inhalten des Gedichts abbildet oder veranschaulicht, daß die Musik etwas vom Text einfängt bzw. etwas von ihm ausdeutet. Die petitio principii läuft Gefahr, auf eine bloße Verdoppelung dieser Inhalte in der Musik hinauszulaufen. Die zweite folgt der Annahme, daß Strukturen der Sprache abgebildet werden, daß sozusagen der Sprachkörper den gemeinsamen Nenner von Text und Musik bildet; diese Annahme begegnet zwischen der Hypostasierung von "Intonationen" bis hin zu musik-sprachlichen Übereinstimmungen (wie etwa bei Thr. G. Georgiades), wobei von vornherein nicht feststeht, ob es sich nicht bloß um Analogien handelt, und in der Regel das "Nationalsprachliche" - was immer es sei - Grund und Ziel des Unterfangens bildet. Damit steht wenn nicht das Allgemeine, so doch das Besondere im Vordergrund, während das Einzelne - gerade dieses Klavierlied von Schubert, Wolf oder wem immer - eher am Rande figuriert und leicht aus den Augen gerät. Die dritte Strategie geht von einer Abbildung der formalen Strukturen eines jeweiligen Gedichts in der Musik aus. Wenn die Form-Strukturen im konkreten Fall jedoch von untergeordneter Bedeutung sind, dann bereitet diese Strategie notwendig Verlegenheit. Bei Heine-Gedichten ist dies stets wieder der Fall, vielleicht am wenigsten in den "prosaischen" Nordsee-Gedichten, unabweisbar jedoch in einem Gedicht wie "Ich stand in dunkeln Träumen".

Vertonungen Heinescher Gedichte im 19. Jahrhundert spielen sich auf einer Skala ab, die gewissermaßen von zwei Extremen begrenzt wird. Nichts zeigt dies deutlicher als die "Lorelei", wie sie einerseits von Friedrich Silcher, andererseits von Franz Liszt in Töne gekleidet worden ist, und zwar räumlich nicht weit voneinander entfernt und fast zur selben Zeit, mithin unter denselben historischen Rahmenbedingungen um $1840^{3}$. Silcher schreibt eine Art schlichtes Volkslied, das dem Aufbau bzw. der Form des Gedichts penibel folgt. Er läßt - ohne Rücksicht auf den Inhalt - Heines Strophen hersingen. Liszt hingegen nimmt das Gedicht zum Anlaß für eine Gesang-Szene oder symphonische Miniatur (eine kleine "symphonische Dichtung"), er komponiert über die Verse, über die Form des Gedichts tendenziell hinweg, um das, was in ihm geschieht, einzufangen. Er wendet sich dem in den Versen enthaltenen Drama zu, das Silcher in Moritaten-Manier gänzlich dem gesungenen Vortrag der Worte überläßt. Fast mag es - zugespitzt gesagt - so scheinen, als könnte bei Liszt, ganz anders als bei Silcher, die Singstimme sogar fehlen, ohne die Aussage der Musik zu schmälern. Konsequenterweise hat Liszt

3 Vgl. ausführlich dazu vom Verf., Heines "Lorelei" in den Vertonungen von Silcher und Liszt, in: Archiv für Musikwissenschaft XLVIII, 1991 , S. $169-198$ 
sein eigenes Lied gleich mehrfach zu einem "reinen" Instrumentalstück umgearbeitet. Er beansprucht durchaus, das wörtliche Geschehen von Heines Lorelei durch Töne allein zu repräsentieren. Während Silchers Melodie keinerlei Bezug zum Inhalt des Heineschen Gedichts aufweist, wohl aber Heines Gedicht als Gedicht in sich aufnimmt, läßt Liszts Klavierlied kaum mehr einen Bezug zu Heines Gedicht als sprachlichem Kunstwerk, wohl aber zu der von Heine erzählten Geschichte erkennen. Größere Gegensätze der Lektüre oder Analyse des Gedichts zum Zwecke der Schaffung eines Lieds sind in jener Zeit des Vormärz schwer vorstellbar.

Es läßt sich nicht in ein paar Worten skizzieren, was ein Heine-Gedicht ist, zumal da die Formen vielfältig sind und die Exemplare überaus unterschiedlich ausgefallen sind. Angedeutet sei nur, daß in den meisten seiner Gedichte, und seien sie noch so kurz, die "Inhalte", voran Handlung und Erzählung, das Entscheidende zu sein scheinen. Ohne die Spezifik der Inhalte und Erzähltechniken läßt sich wohl kaum wirklich von einem Heine-Gedicht sprechen. Hingegen reicht ein Abzählen der Verse, Untersuchen der Metren und Zusammensuchen der Reime an Heine noch weniger heran als an andere Dichter seiner und der nachfolgenden Generation. Mit einem allein formellen Vorgehen ist bei ihm nicht allzuviel auszurichten, und auch die im 19. Jahrhundert entstandenen Lieder nach Heine versprechen nicht allein musikgeschichtlichen Aufschluß, sondern sind darüber hinaus geeignet, Einblicke zu gewähren in die Verfassung der bürgerlichen Epoche insbesondere des deutschsprachigen Raumes in dem Säkulum zwischen dem Wiener Kongreß und dem Ersten Weltkrieg.

Es steht zu vermuten, daß die Dominanz von Inhalt, Handlung und Erzählung dazu beigetragen hat, daß Heines Gedichte sich trotz ihrer immensen Popularität, die freilich von Anbeginn an von Mißverständnissen begleitet gewesen sein mag, mit der Zeit etwas abgenutzt haben. Auch die schwindende Popularität könnte im Verein mit der so spürbaren Dominanz der "Inhalte" die Attraktivität von HeineGedichten für die erste Garde der Liedkomponisten gemindert haben. Schließlich dürfte es nicht zufällig sein, daß die bitteren, bissigen, bösen Heine-Gedichte weder zur Mitte noch zum Ende des Jahrhunderts zahlreich vertont worden sind; sie scheinen als lyrische Kabinettstücke schon auf das Kabarett vorauszuweisen, ohne sie damit verkleinern zu wollen, sofern das Kabarett offenbar unausweichlich mit "Kleinkunst" assoziiert wird. Der schöne Schein, nach dem die Bürger sich besonders in der Musik sehnten, hatte für den politischen Dichter, das Politikum Heine wenig Verwendung. Für Klavierlieder zwischen Mendelssohn, Schumann und Brahms wurden die frühen, die "romantischen" Gedichte Heines deutlich bevorzugt, - ganz unabhängig von der Frage, ob Heine nicht auch in ihnen (wie in der "Lorelei") das romantisch Gefühlvolle durchschaut und durchbrochen hat. Jedenfalls dominierten in den Kompositionen jene Gedichte, die Stimmung und 
Gemütlichkeit trafen, die von Herz und Schmerz sangen. Fast scheint es so, als sei am raschesten dort bei Heine zugegriffen worden, wo wichtige Dimensionen seiner Gedichte gar nicht vorhanden sind. Der schwere Stand, den Heine dann gerade unter Künstlern und Intellektuellen im 20. Jahrhundert weithin hatte, dürfte mit diesem "Verständnis" zu tun haben. Wer Gedichte etwa von Baudelaire, Rilke oder Stefan George als Kunst-Leitsterne im Herzen trug, für den war - noch abseits von allen anderen zeittypischen Komplikationen wie dem Antisemitismus - die Gedichtkunst Heines etwas, über das zu überheben man sich leicht, vielleicht allzuleicht berechtigt glaubte.

Die Strukturen, die ein Heine-Gedicht also besonders auszeichnen - vereinfacht gesagt der Inhalt, die Handlung und die Erzähltechnik -, müssen der Musik weitgehend unzugänglich bleiben, zum Beispiel Träume, Tagträume, Einbildungen und alle anderen Verwerfungen zwischen Realität und Irrealität, für deren Schilderung in ihren Graden die poetische Darstellung so geeignet ist. Aber gerade das, worauf der Reiz eines Gedichts am meisten beruht, läßt sich oft am wenigsten in Töne einfangen. Das gilt auch für die Verse "Ich stand in dunkeln Träumen" und ihr kompliziertes Geflecht aus Traum und Wirklichkeit, von dem nicht leicht anzugeben ist, in wie vielen Verschlingungen es anzutreffen ist ${ }^{4}$ :

Ich stand in dunkeln Träumen

Und starrte ihr Bildniß an,

Und das geliebte Antlitz

Heimlich zu leben begann.

5 Um ihre Lippen zog sich

Ein Lächeln wunderbar,

Und wie von Wehmuthsthränen

Erglänzte ihr Augenpaar.

Auch meine Thränen flossen

10 Mir von den Wangen herab -

Und ach, ich kann es nicht glauben,

Daß ich dich verloren hab'!

Strophenbau, Versbau, Metrik und Rhythmik sind so gewöhnlich und wenig aufschlußreich, daß man dem, was ein Heine-Gedicht ausmacht, in diesen Dimensionen kaum begegnet. Das Zentrum des Gedichts bildet die Beziehung des

4 Die Wiedergabe des Gedichts folgt Bd. I,1 der von M. Windfuhr bei Hoffmann und Campe herausgegebenen Historisch-kritischen Geamtausgabe der Werke Heines (gleichlautend in den beiden dort S. 232/234 und 233/235 abgedruckten Versionen). 
Ich zu einem imaginierten Du. (Nur bei Schubert ist dem titellosen Gedicht eine durchaus sinnvolle Überschrift gegeben: "Ihr Bild".) Das Arrangement hierfür ist kunstvoll: "Ich" steht gebieterisch am Anfang, kehrt aber erst in der dritten Strophe wieder (Z. 9: "meine"; Z. 10: "mir"; Z. 11 und 12 "ich"), während inzwischen - indirekt - von "ihr" und "ihre" die Rede ist (Z. 2, 5 und 8), ehe die Schlußzeile sowohl die direkte Anrede als auch die gewünschte, zugleich aber als (eingebildeter) Verlust begriffene Vereinigung in der Pointe "ich dich" vorführt. Nicht weniger ausschlaggebend ist die Folge der Verben, die einerseits verschiedene Bewegungsarten anzeigen und die andererseits in unterschiedlichen Tempora verwendet sind. Bis Zeile 9 lassen sechs Verben einen Prozeß erkennen, in dem sich Stillstand über Starre in Bewegung auflöst, mehr und mehr liquidiert wird: "stehen", "starren", "zu leben beginnen", "sich ziehen", "erglänzen", "fließen". Bis hierher ist das Präteritum die einzige Zeitform. Zu Beginn des letzten Zweizeilers durchgängig bilden Zweizeiler die inhaltlichen Einheiten - springt Heine, wie er es liebt, in gewisser Weise aus dem Gedicht heraus mit einem Verbum ganz anderer Qualität und im Präsens ("ich kann es nicht glauben"), ehe alles definitiv der Vergangenheit überantwortet wird (nun im Perfekt: "ich dich verloren hab'"). Eigentlich alles das, was dieses Gedicht als Gedicht preziös macht, muß bei der Umsetzung in Musik (jenseits des Vortrags der Worte, sofern er verständlich bleibt) unter den Tisch fallen, voran das Ich-Du-Verhältnis und die Zeitformen; am ehesten lassen sich prinzipiell die Bewegungsformen kompositorisch einfangen. Der Komponist muß die Klippe umschiffen, die sich dadurch auftut, daß er das Zentrum des Gedichts nicht erreichen kann und dennoch sein Klavierlied schreiben muß.

\section{Schubert und Wolf im Vergleich}

Die folgenden Bemerkungen zum Vergleich der Lieder von Schubert und Wolf nach Heines "Ich stand in dunkeln Träumen" sollen, ohne die Lieder bewerten und gegeneinander aufrechnen zu wollen - nur auf einige Ähnlichkeiten und Unterschiede des Umgangs mit der Vorlage hinweisen und dem jeweiligen Grundzug der beiden Lied-Kompositionen folgen. Eine vollständige Analyse der Lieder ist weder beabsichtigt noch in Kürze möglich.

Es scheint so, daß Schubert in seinem Vorspiel stockend, schleppend anfängt und damit von der Statik ausgeht, von der am Gedichtbeginn die Rede ist, während Wolf gleich mit der Bewegung einsetzt, in die das Gedicht erst allmählich übergeht, - zwei Lesarten, von denen jede etwas für sich hat (Beispiel $1^{5}$ ).

5 Wir folgen im Falle von Schubert der 1976 von D. Fischer-Dieskau und E. Budde bei Litolff/Peters in Frankfurt a.M. besorgten Ausgabe des Schwanengesangs (Edition Peters 8302a, S. 36f.), im Falle von Wolf dem $1980 \mathrm{im}$ Musikwissenschaftlichen Verlag Wien von H. Jancik vorgelegten Bd. VII,1 (S. 7ff.) der Sämtlichen Werke. 
(a)

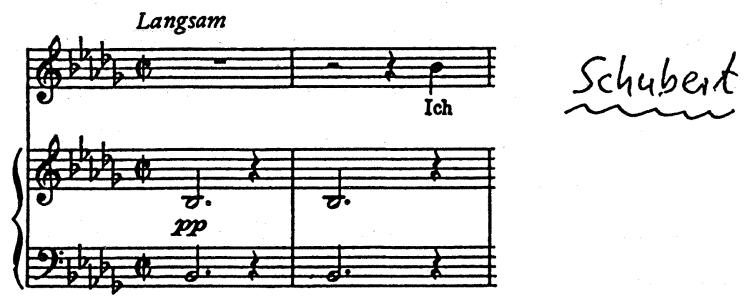

(b)
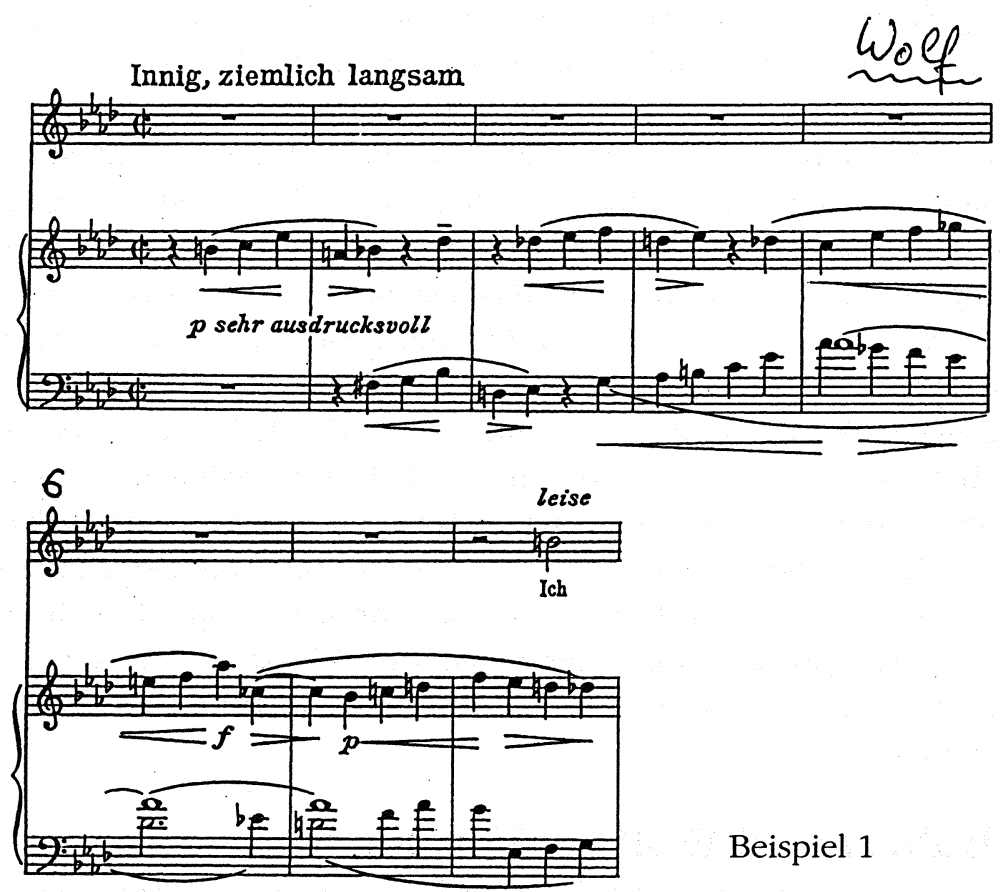

Beispiel 1

Die zwei Töne, aus denen Schuberts Vorspiel besteht ${ }^{6}$, sind kaum mehr als eine Intonationshilfe für den Sänger und doch in aller Unscheinbarkeit eine, wenn nicht die kompositorische Zelle für das gesamte Lied. Beim Einsatz der Singstimme verwendet Schubert sie sogleich wieder. Wolf verfährt ebenso mit dem ersten Motiv den ersten fünf Tönen - seines opulenteren Vorspiels, dessen chromatische Folge charakteristisch ist; die Verwendung chromatischer Vorhalte erweist sich im gesamten Lied als Manier, wobei es schwierig ist zu entscheiden, inwieweit diese Manier für den damaligen Wolfschen Tonsatz typisch oder für dieses Lied speziell charakteristisch ist. Nur ein Schelm wird an der Tonfolge die retrograde Form des 
so bekannten (angeblich "sprechenden", in Wirklichkeit abstrakt symbolisierenden) b-a-c-h-Motivs herauslesen und einer Analyse des Liedes zugrundelegen wollen, obwohl der fünfte Ton - der Ton es in der Mitte - weit weniger stört, als es den Anschein haben mag: Erstens ist es ohne methodische Skrupel in den musikanalytischen Zuordnungsspielen höchst gewöhnlich, daß die gesuchten Töne nicht in ununterbrochener Reihenfolge erscheinen, zweitens kann gerade der in der Mitte liegende Ton es denen, die das symbolische Geschäft der Zuweisung von Buchstaben und Tonbezeichnungen lieben, willkommen sein, weil sich mit dem Ton es die Initiale S (für Sebastian) verbinden läßt, - ebenfalls eine allzu geläufige Zuordnung. Unabhängig von einem solchen wagemutig-waghalsigen Symbolismus liegt es näher zu fragen, wozu Wolf mit einer Art zweistimmigem Fugato beginnt, das an eine Invention gemahnt. Ist man nicht versucht, in den beiden Stimmen zwei Personen - das Ich und das Du des Gedichts - zu erkennen? Aber entstünde dadurch nicht ein anderes willkürliches Metaphernspiel oder Symbolisieren, das nicht schon deshalb Plausibilität besäße, weil derlei Zuweisungen und Zuordnungen im musico-semantischen Alltag üblich sind und nicht selten zum Ausgangspunkt für Aussagen über Vokalmusik im allgemeinen und über Klavierlieder im besonderen werden?

Die beiden Lieder von Schubert (b-Moll) und Wolf (As-Dur) sind ungefähr gleich lang, obwohl sie bei fast derselben Takt- und Tempovorschrift (Schubert: alla breve, "langsam"; Wolf: alla breve, "innig, ziemlich langsam") beträchtlich verschiedene Ausdehnung besitzen: 36 Takte bei Schubert und 62 bei Wolf. Wolfs Lied ist deutlich rascher $\mathrm{zu}$ singen. Es ist nicht einfach $\mathrm{zu}$ entscheiden, ob der Hinweis "innig" zu Beginn des Wolfschen Liedes einen Hinweis zur Deutung liefern soll oder liefert. Zu dem, was sich in dem Gedicht dramatisch zuspitzt, paßt er nicht. So gesehen liegt es nahe, "innig" als eine bloße Anweisung zum Vortrag anzusehen, und zwar als eine, die nicht einmal für das Wolfsche Lied selbst zur Gänze gelten kann; die Steigerung und der Höhepunkt gegen Ende des Liedes lassen sich sängerisch nicht "innig" darstellen. Wolf folgt dem Wechsel des Gedichts von anfänglicher Versunkenheit zu Resignation, die er sich, wie es scheint, nicht so sehr als still, sondern als theatralisch ausgreifend vorstellt. So wenig die Vortragsanweisung "innig" hier zu denotieren vermag, so üppig mögen im Umfeld von Stichworten wie "Romantik", "deutsches Lied" usw. die Konnotationen blühen.

Die Disposition der drei Heine-Strophen in den beiden Liedern weist Ähnlichkeiten auf, am Ende jedoch auch gravierende Unterschiede. Die Symbolzeichen des Diagramms (Beispiel 2) sollen nur eine grobe Veranschaulichung des Aufbaus geben und sind gewählt, weil bei Halbkreisen und Dreiecken wohl niemand daran denkt, daß etwas an der Musik getroffen wird, wie es bei den üblichen, nicht weniger arbiträren Buchstabenzeichen (A, B, C; a, b, c usw.) als Repräsentanten 
von musikalischen Formteilen so oft prätendiert wird, ohne daß es auch dort der Fall sein müßte (Beispiel 2).

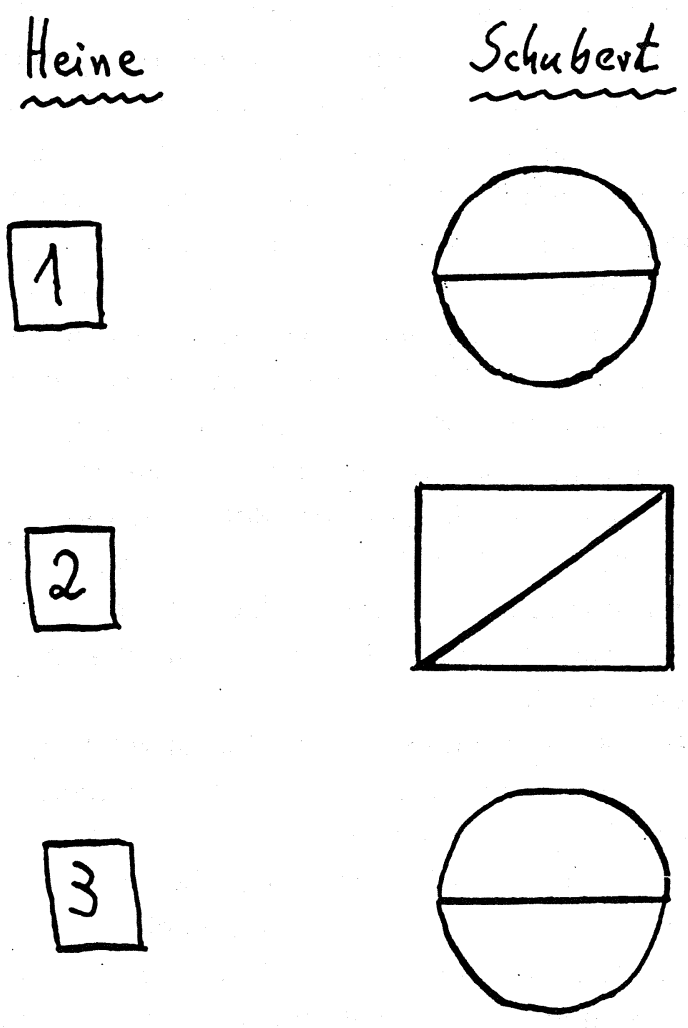

Beispiel 2
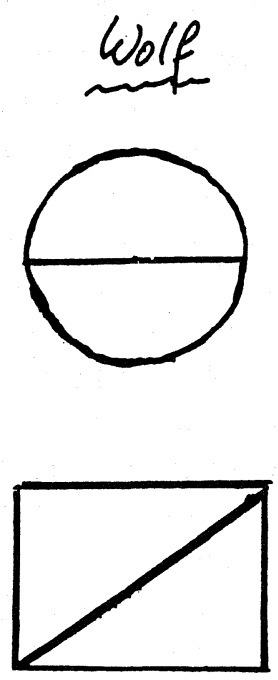

Schubert vertont die erste und dritte Strophe Heines gleich, die zweite etwas anders. Die Halbierungen der Zeichen in dem Diagramm sollen andeuten, daß die für das Gedicht konstitutive Einheit des Verspaares, von denen jede Strophe zwei aufweist, gewahrt bleibt. Zwar beinnt auch Wolf die dritte Strophe reprisenartig, aber anders als Schubert weicht er dann davon ab. Während Schubert im gesamten Lied, auffällig genug, auf die Beigabe jeder Textwiederholung verzichtet, läßt Wolf in der Höhepunktbildung seines - anders als bei Schubert: final ausgerichteten - Liedes das letzte Verspaar zweimal vortragen ${ }^{7}$. Darauf wird noch zurückgekommen werden.

7 Sowohl Schubert als auch Wolf folgen streng dem Heineschen Wortlaut; Abweichungen sind so minimal, daß sie unberücksichtigt bleiben können, z.B. bei Schubert - wohl um die rhythmische Idee der doppelten Punktierung verwirklichen zu können - "starrt" statt "starrte" in Z. 2 und bei Wolf "ich kann's" statt "ich kann es" in Z. 11. 
Wenden wir uns zunächst dem Einsatz der Singstimme bei beiden Komponisten zu (Beispiel 3).

(a)

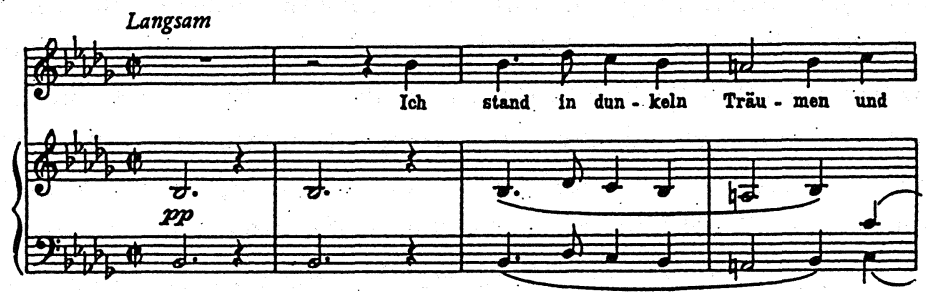

Schubert

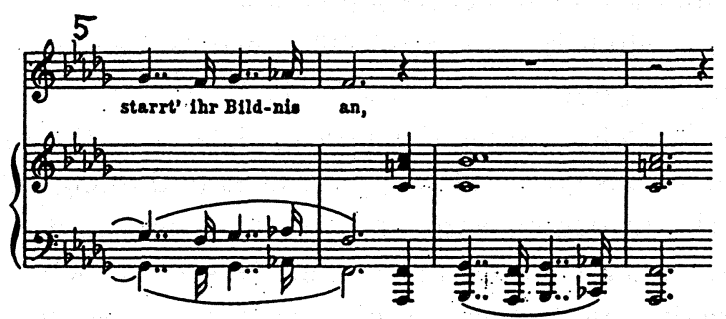

(b)
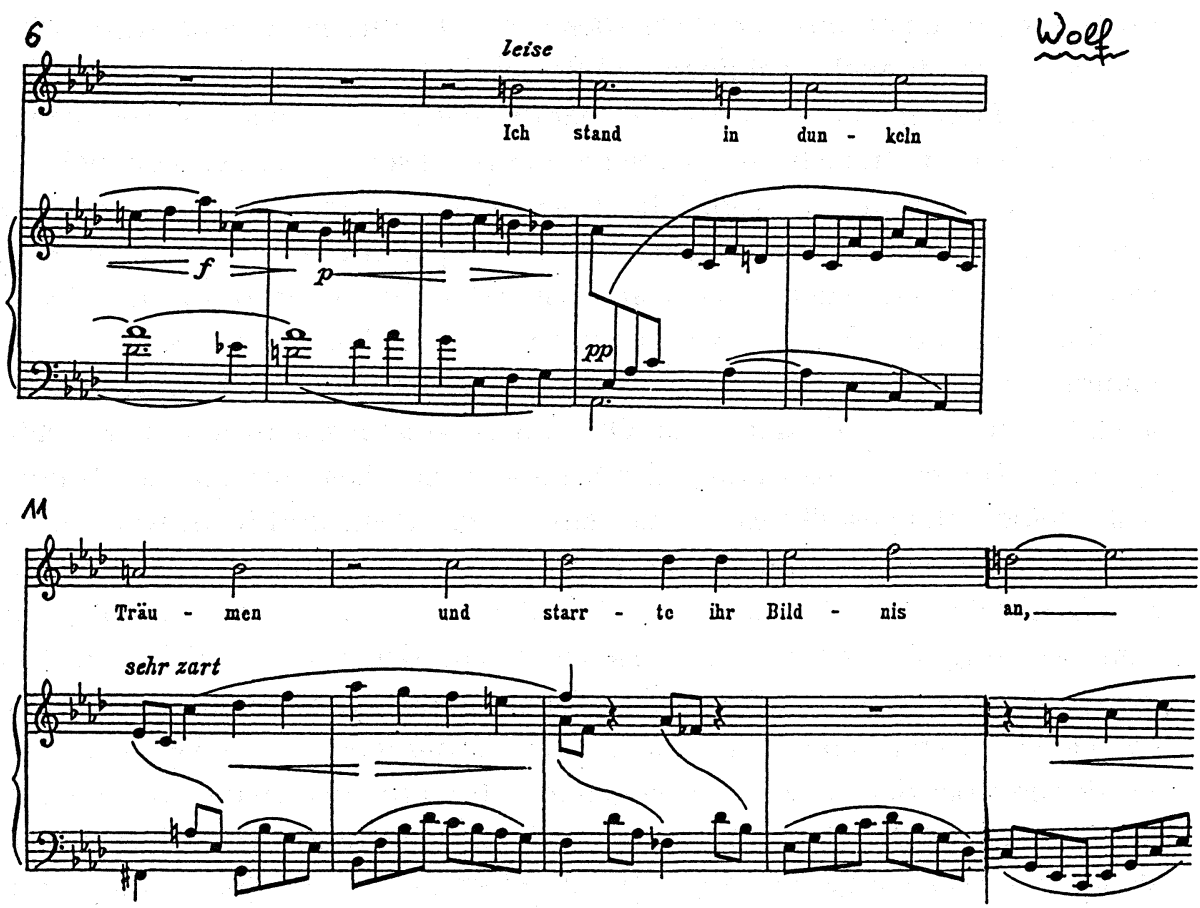

Beispiel 3 
Schubert beginnt einstimmig, unisono. Erst nach Vortrag des ersten Verspaares geht die Klavierbegleitung wie ein Echo in einen akkordischen Satz über. Die beiden fast unspezifischen, elementarsten Töne des Vorspiels werden, wie es solchen Zellen in der Musik gewöhnlich ergeht, durch rhythmische, melodische und damit auch harmonische Modifikationen entfaltet bis hin zu den auf- oder abtaktig zu verstehenden doppelten Punktierungen, die im weiteren Verlauf des Liedes noch in Varianten begegenen werden. Als zweites winziges Element tritt - bei "stand in" - das Intervall der kleinen Terz b' - des" hinzu, das allerdings streng genommen ebenfalls als melodische Modifikation der beiden Anfangstöne $b-b$ angesehen werden kann, sofern sich unter Zuhilfenahme von Modifikationen, Umkehrungen, Spiegelungen usw. fast alles auf dieses Partikel beziehen läßt.

"Der Text wird eher rezitiert als wirklich gesungen." Gilt diese Anmerkung Dieter Schnebels zu Schuberts "Doppelgänger" nicht auch dafür, wie Schubert die Singstimme in "Ich stand in dunkeln Träumen" einsetzen läßt? Die Bemerkung stimmt nachdenklich, weil sie voraussetzt, daß es eigentliches und uneigentliches Singen gibt. Herrscht demnach am Beginn von Schuberts Lied ein musikalischer Mangel? Während andere wie Gerald Moore mit feinem Gespür in Schumanns ähnlich "rezitierend" beginnendem und ähnlich tonkargem Heine-Lied "Ich hab" im Traum geweinet" aus der Dichterliebe das schwesterliche Pendant zu Schuberts "Ich stand in dunkeln Träumen" erblickten, schlagen wir hier die Brücke von Schuberts Beginn zu dem von Wolf über denselben Text. Wie Schubert verwendet Wolf die bei ihm freilich umfangreichere musikalische Zelle des Vorspiels für den Einsatz der Singstimme. Vielleicht wäre es in beiden Fällen angemessener zu sagen, daß der Einsatz der Singstimme die Hauptsache ist, die im Vorspiel als der Nebensache wiederverwendet wird; das Hysteron-Proteron ist der Bedeutung nach keines. Die Differenz zwischen zwei und fünf Tönen einer solchen Zelle hat natürlich Konsequenzen sowohl für den kompositorischen Umgang mit ihr als auch für die Wiedererkennbarkeit. Ob man in Wolfs Insistenz auf die Halbtöne $\mathrm{h}$ $\mathrm{c}$ und $\mathrm{a}-\mathrm{b}$ eine Intensivierung des Schubertschen Leittonschritts $\mathrm{a}-\mathrm{b}$ erblicken soll, bleibe dahingestellt; es könnte dieses nicht mehr und nicht weniger zufällig sein als die Übereinstimmung der kleinen Terz c - es bei Wolf mit b - des bei Schubert. Die Deklamation bei Wolf und Schubert ähnelt sich auffällig; an der Verdoppelung der Notenwerte im Lied von Wolf darf man sich dabei nicht stören, da sie, wie gesagt, durch den realen Tempounterschied nivelliert wird. So wenig die vielen Übereinstimmungen oder doch wenigstens Ähnlichkeiten der beiden Versionen trotz der dazwischen liegenden 50 Jahre zu verwundern brauchen, so deutlich besteht der Unterschied in der Klavierbegleitung, die Wolf sehr viel selbständiger - insbesondere in Ansätzen zu selbständigen Gegenstimmen - führt.

8 D. Schnebel, Eine Depression und ibre Auflösung. Schuberts Heine-Lied "Der Doppelgänger", in: Von Dichtung und Musik. "Heinrich Heine". Ein Lesebuch, Tutzing 1995, S. 133. 
Das zweite Verspaar - "und das geliebte Antlitz heimlich zu leben begann" - arrangiert Schubert als harten, starken Kontrast in vielen Dimensionen des Tonsatzes; derselbe Kontrast begegnet natürlich in der identisch komponierten dritten Strophe. Die Prozedur gleicht beispielsweise der im ersten Lied der Winterreise dort, wo dem anfänglichen, in der Melodie herb klingenden "Fremd bin ich eingezogen, fremd zieh' ich wieder aus..." rasch das melodisch einschmeichelnde "das Mädchen sprach von Liebe, die Mutter gar von Eh'..." entgegengesetzt wird." An die Stelle des Unisono bei "Ich stand in dunkeln Träumen..." tritt bei "und das geliebte Antlitz..." ein voller Satz (fast eine Art "Choralsatz") mit Kadenzharmonien, erklingt statt des Beginns in Moll die Dur-Variante und wird, wenn man so sagen darf (ohne nachdenklich zu werden), höchst sanglich gesungen (Beispiel 4).
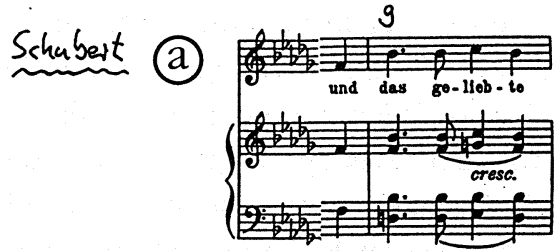
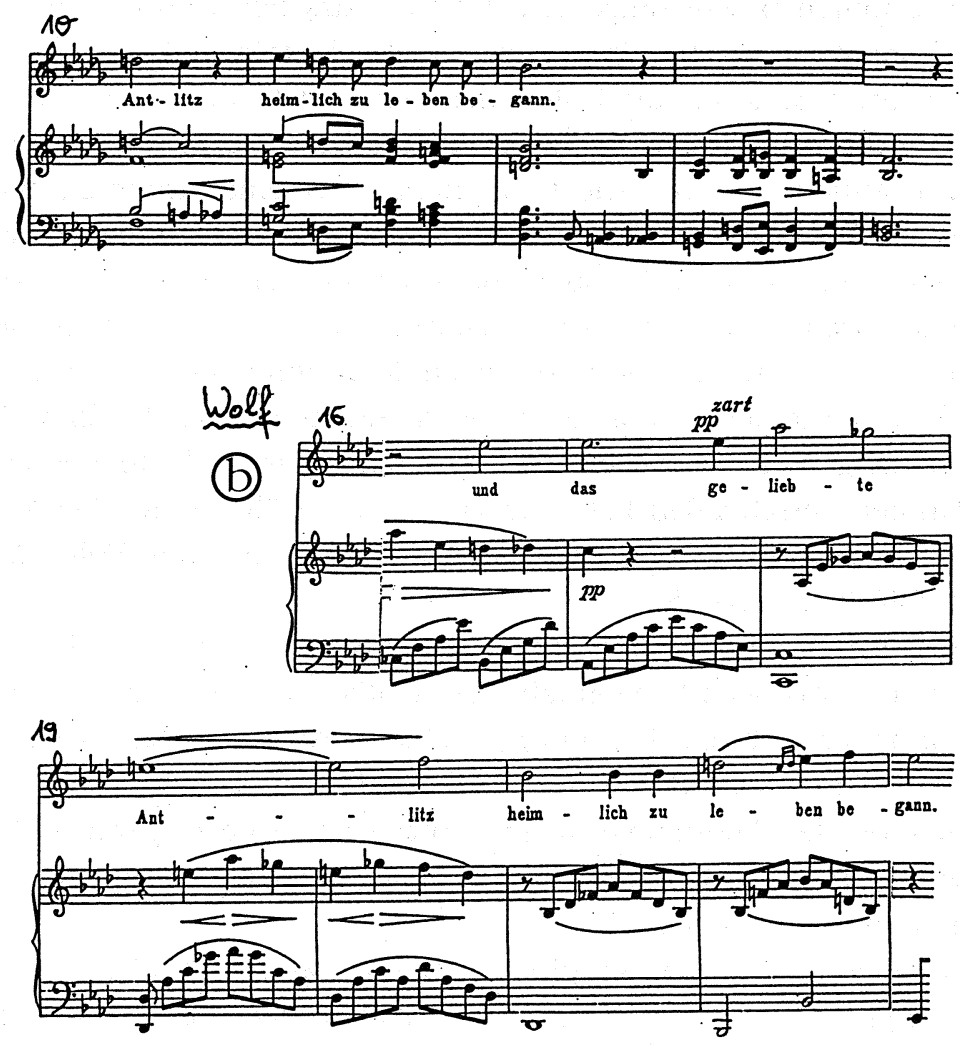

Beispiel 4 
Im zweiten Verspaar entspricht die Deklamation bei Wolf (in doppelten Notenwerten) der bei Schubert noch genauer. Aber schon als Konsequenz des bewegteren Vorspiels und der freieren, gelenkigeren Begleitung ist für Wolf hier kein größerer Kontrast erforderlich. Während Schubert eigentlich nirgendwo hin will, versucht Wolf Bewegung hinzuzugewinnen, will er quasi Raum und Zeit öffnen, sucht er so etwas wie Entwicklung in einem einheitlichen Zug an Bewegung. Die Klavierbegleitung bleibt wie zuvor gegenüber der von Schubert freier, aber sie hält sich noch deutlich im Hintergrund. Erst im weiteren Verlauf des Wolfschen Liedes ändert sich dies; auch das gehört zu dem einheitlich-vereinheitlichenden Vorgehen, das bei Schubert im Bereich kleiner Motive (Zellen) zu erkennen ist, bei Wolf jedoch den Duktus des ganzen Liedes ergreift.

Obwohl Schubert die erste und die dritte Strophe, deren Inhalte unvergleichlich sind, gleich vertont, hält er sich enger an den Text, sofern er den Zweizeilern folgt und für jeden, deutlich abgesetzt, einheitlich vier zusammengehörige Takte reserviert, auch in der zweiten Strophe, in der die beiden Verspaare grosso modo dieselbe Musik erhalten. Zwar ist letzteres bei Wolf nicht anders, aber dieser verkürzt nun, gewissermaßen um Zeit zu gewinnen, in der zweiten Strophe die Notenwerte. Dadurch beschleunigt sich das Lied, das bei Schubert eher schleppend vorankommt, wozu bei ihm nicht zuletzt beiträgt, daß er die beiden Vorspielnoten (b) nun zu breiten Haltetönen verwendet bzw. ausbaut (Schubert, T. 15f.), wobei die dem Gesang nachfolgende markante Sechzehntel-Auftaktfigur mit ihrer Terzstruktur (T. 18) leicht erkennbar aus einer früheren Wendung abgeleitet ist (T. 5f., T. 7f.; vgl. Beispiel 3a), wenn man sie nicht gleich als rhythmische Variante der beiden Vorspieltöne oder der ersten beiden Töne der Gesangstimme ansehen möchte. Man ist natürlich versucht, diese markante Figur auf irgend etwas an dem Gedicht zu beziehen, aber ohne den Schlüssel, den nur der Komponist selbst liefern könnte, steht zu fürchten, daß ihr Sinn sich nur gedichtunabhängig und musikalisch - als Zusammenhang schaffendes kompositorisches Partikel - erschließen läßt. Die Deklamation ähnelt sich bei Wolf und Schubert zwar erneut, aber durch die neuen Notenwerte gewinnt Wolfs Lied erheblich an Flexibilität (Beispiel 5).

In der zweiten Strophe holt Wolf (wenn man so sagen darf) weiter auf, ja er überholt Schubert: Die Wolfsche Version fällt nun deutlich kürzer als die Schubertsche aus. Bei Wolf ändert sich die Begleitung: Triolen werden eingeführt (ein weiteres Mittel zum Evozieren von Beschleunigung), und doch tritt sie weiter zurück, sofern im Klavier der Singstimme auch ansatzweise keine Gegenmelodie mehr zugesellt wird (eine Melodiestimme begegnet im Klavier jetzt nur noch in den Pausen zwischen den Verspaaren).

Während Schubert die zweite Strophe als einen von den beiden gleich komponierten Außenstrophen unterschiedenen Mittelteil setzt, dient diese Strophe 
(a)
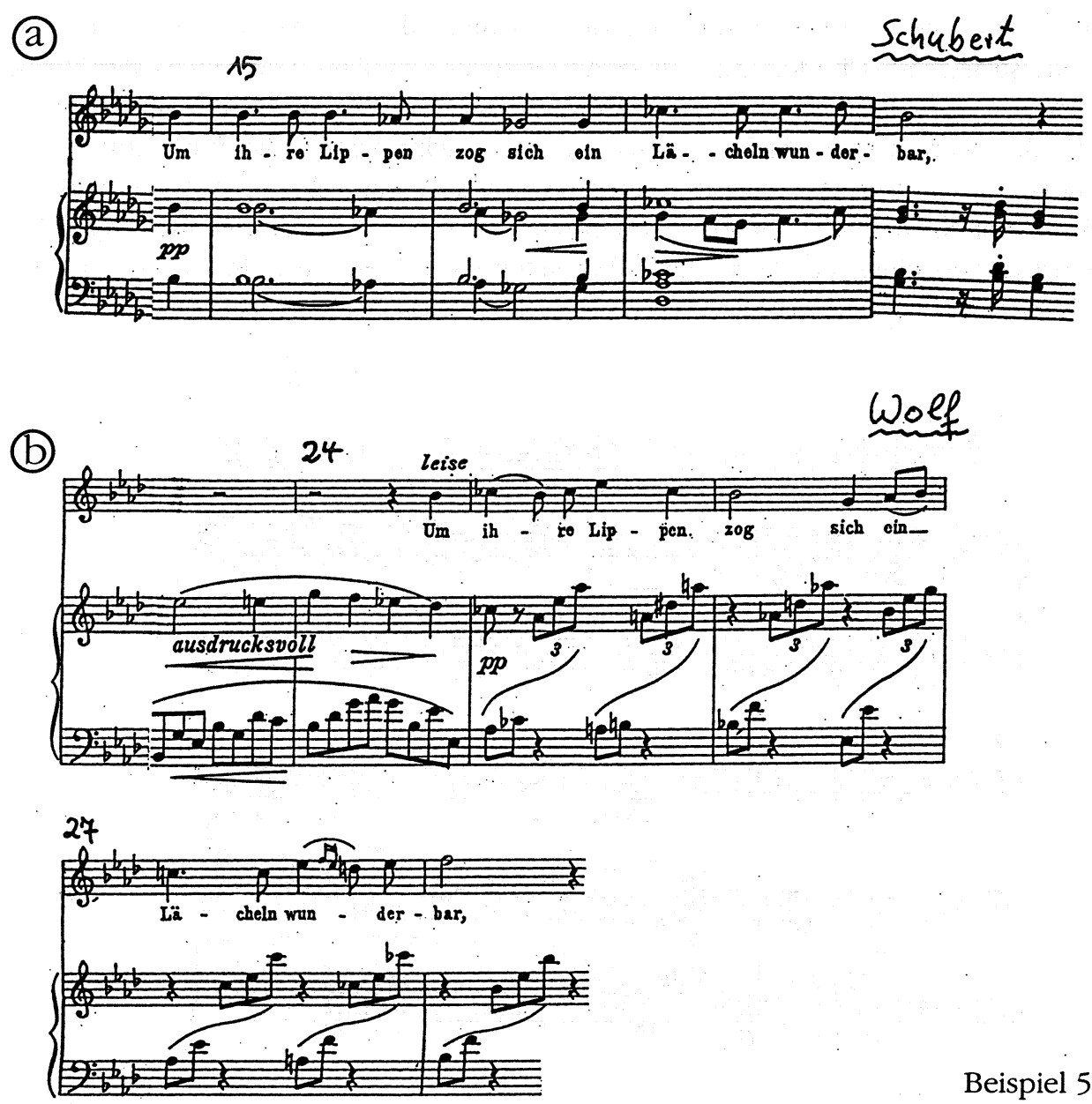

Beispiel 5

Wolf als Durchgangs- oder Überleitungsteil, als Vorbereitung zur dritten, der sein Augenmerk vorzugsweise gilt und die ihrerseits zusammen mit dem Nachspiel ungefähr so lange dauert wie das - analoge - Vorspiel und die ersten beiden Strophen zusammen. In nichts weicht er von Schubert so sehr ab wie darin. So wenig Mühe Schubert sich mit dem "Vorspiel" gemacht hatte, so unscheinbar bearbeitet er das "Nachspiel": Er nimmt die beiden der ersten Strophe folgenden Takte der Klavierbegleitung (T. 12f.; vgl. Beispiel 4a), wendet sie von B-Dur nach b-Moll und läßt sie forte statt piano vortragen. Nichts sonst weist darauf hin, daß im Fortgang des Gedichts ein höchst schmerzhafter Prozeß vollzogen worden ist. Vielleicht wollte Schubert uns bedeuten, daß sich das Ganze nur in der Einbildung des lyrischen Ich abspielt und sich im Verlauf des Gedichts in Wirklichkeit nichts verändert hat. Das jedoch bleibt bloße Spekulation der Auslegung. 
Wolf inszeniert hingegen seinen großen Schluß als Ziel, vorbereitet durch die Mittelstrophe als Überleitung. Das letzte Verspaar veranlaßt eine Klimax der Musik bzw. wird in sie hineingenommen, wird wiederholt und erreicht in dieser Wiederholung an der Stelle, an der die Uneinsichtigkeit in die Wirklichkeit formuliert wird ("ich kann's nicht glauben"), einen Höhepunkt, der trugschlüssig (fMoll) anhebt und über einen mehrdeutigen verminderten Septakkord fortgeführt wird, um in einen das Fortissimo überbietenden Akzent zu münden (T. 50 - 52, Beispiel 6).
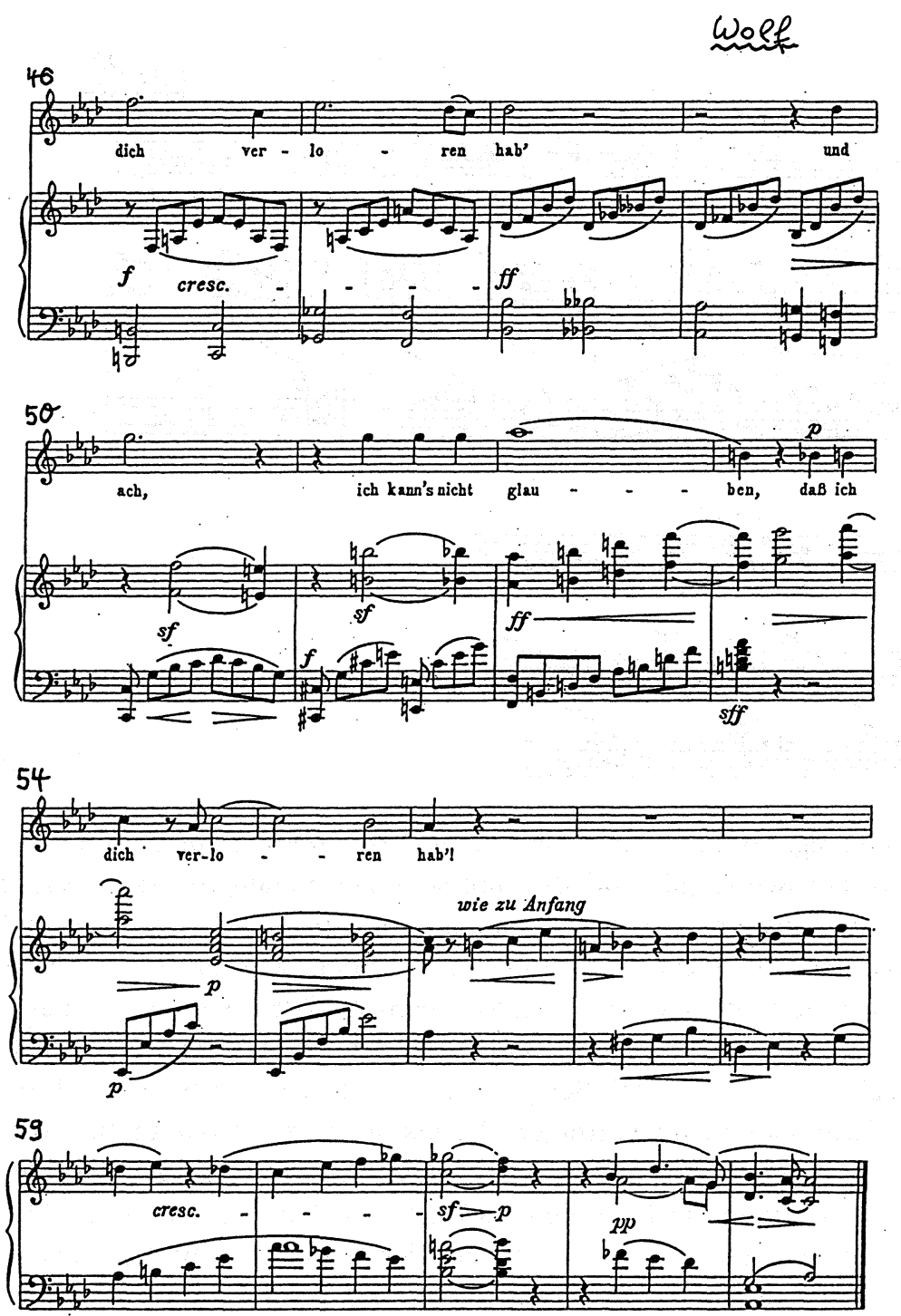

Beispiel 6 
Mit dem nicht zuletzt in der Wiederholung des letzten Verspaares kulminierenden Zielpunkt zeigt Wolf sich weit von dem Schubertschen Konzept entfernt. Doch die ins Grandiose gehende Geste ist nicht weniger prekär als die Wiederholung des Verspaars selbst. So approbiert, schon weil omnipräsent, das Mittel sein mag, Worte, Verse und insbesondere Schlußwendungen in der Arien- und Liedkomposition zu wiederholen, so empfindlich stört, wenn nicht zerstört die Prozedur das Gedicht als Kunstgebilde gerade bei einem Schriftsteller wie Heine, der in seinen Versen Wiederholungen bewußt, gezielt und sparsam einsetzt. Wenigstens dem Gedicht, das als Kunstprodukt Anspruch darauf hat, unversehrt zu bleiben, wird durch wiederholende Verfahren Gewalt angetan. Zwar scheint in vielen Klavierliedern des 19. Jahrhunderts das Eigengewicht des Gedichts bzw. der Verse mehr respektiert worden zu sein als früher, aber der Respekt ging von Schubert bis Mahler und von Schumann bis Wolf keineswegs so weit, daß er vor der Willkür der Wiederholungen (im positivsten Falle wie hier bei Wolf im Dienste der musikalischen "Dramaturgie", im gewöhnlichen als bloße Usance) haltgemacht hätte.

Schubert legt auf den Vortrag des Gedichts und auf dessen Strophenbau Gewicht, er verwendet minimale musikalische Mittel und läßt Heine den Vortritt. Wolf akzentuiert anders. Er beansprucht, die im Gedicht geschilderte Geschichte mit musikalischen Mitteln selbst zu erzählen, er arbeitet auf ein Ziel hin und setzt sich dabei mehr als Schubert über das Gedicht qua Gedicht hinweg, um den Inhalt besser zu treffen. Wolfs Analyse des Gedichts geht auf die Stelle zu, an der Heine in den beiden Schlußversen aus der bisherigen, in der Vergangenheitsform gehaltenen Schilderung heraustritt ("ich kann es nicht glauben"), - eine Wendung der Erzählung, die Schubert offenbar völlig ignorieren kann. Diese Beobachtung läßt sich formulieren, ohne schon in die Gefahr zu geraten, die deutende Behauptung aufzustellen, daß Schubert in seinem Lied von Anfang bis Ende eine an Heine abgelesene Ausweglosigkeit des Geschehens treffen bzw. wiedergeben möchte (was möglich, aber nicht "beweisbar" ist).

Sieht man von der Klimax ab, die Wolf für die dritte Strophe arrangiert, so verblüfft die Übereinstimmung der Deklamation mit der von Schubert, wenngleich sie differenzierter und flexibler ist. Wolfs effektvolle melodische Linie ist durchaus einfach zu singen, seine Klavierbegleitung aufwendiger und selbständiger als die von Schubert. Auf der genannten Skala des extrem gegensätzlichen kompositorischen Umgangs mit einer Heineschen Vorlage steht Wolf mit "Ich stand in dunkeln Träumen" bestimmt näher bei Liszt, Schubert wiederum näher bei Silcher, wiewohl Schubert seine Lieder - auch und besonders "Ich stand in dunkeln Träumen - so viel raffinierter komponiert hat als Silcher. Bei diesem kommt alles auf die Melodie an und nichts auf die Begleitung, der Schubert so große Aufmerksamtkeit schenkt. Wenn eine Silchersche Melodie ohne Begleitung gesungen wird, ist nahezu das 
gesamte Lied vorhanden, wenn Schuberts Lied ohne sie gesungen wird, dann hat man nur einen Bruchteil von ihm, den man anders als im Falle Silchers nicht selbst zum Ganzen zusammensetzen kann. Mit seinem auf Entwicklung zielenden, "dramatischen" Lied rückt. Wolf hingegen eher in die Nähe Liszts, der seinerseits für seine deutschsprachigen Lieder auch an der französischen Vokalkomposition Orientierung gefunden haben dürfte. Anteil haben bei Wolf daneben natürlich alle jene kompositorischen Möglichkeiten, die in dem halben Jahrhundert zwischen Schubert und ihm ausgebildet worden sind. Wolf kann auf Schuman und viele andere aufbauen, die für die mitteleuropäische Liedkomposition aktuelle Mittel bereitgestellt haben.

Ein Gedicht, zumal eines von Heine, muß in der Komposition eines Klavierlieds vielleicht weniger "erklärt", "gedeutet", "interpretiert" als vielmehr inszeniert werden. Die Vorlage lieferte Heine, nicht aber das Drehbuch, das einmal Schubert, das andere Mal Wolf geschrieben haben, - beide als Regisseure für musikalische Filme, die unterschiedlich ausgefallen sind, weil jeder Stoff eine unbestimmtunbestimmbare Zahl an Drehbüchern ermöglicht. In den Verwertungszusammenhängen des Vertonens muß wie in denen des Verfilmens an den Vorlagen gearbeitet, modelliert, improvisiert werden, und zwar gerade auch dann, wenn es sich um Vorlagen eigenen und womöglich hohen Kunstanspruchs handelt. Leider kennen wir von den Klavierliedern des 19. Jahrhunderts in aller Regel nur die Vorlage und das fertige Produkt, nicht auch das Drehbuch als den Umschlagplatz, in dem sich die Analyse der Vorlage am klarsten spiegelt.

Im Ergebnis sind die beiden Klavierlieder nach Heines "Ich stand in dunkeln Träumen" trotz ihrer Unterschiede verwandt. Wolfs Version ist ohne Schuberts Lied kaum denkbar. Man braucht sich nicht zu scheuen, den Wolfschen Versuch ein musikalisches Remake zu nennen, um in den Bildern der Sprache des Films zu bleiben. Das meint alles andere als einen Abklatsch, und es ist keineswegs despektierlich gemeint, im Gegenteil: Je nach Kenntnis, Geschmack und Urteilsparameter läßt sich das eine mehr als das andere schätzen, ja manche möchten vielleicht sogar das Remake für das Original halten. Zwischen Schubert und Wolf hatte sich sozusagen die Filmtechnik geändert, wie es binnen 50 Jahren auch erwartet werden darf. Der junge Wolf freilich hatte nicht nur Heines Vorlage vor Augen, sondern auch Schuberts Komposition im Nacken, - Last und Herausforderung in einem.

Wie dem Cineasten bei einem Remake wird es auch dem Musikliebhaber möglicherweise mehr Vergnügen bereiten, die Wahl zwischen beiden Versionen über denselben Stoff zu haben, als jede der Versionen für sich an der Vorlage zu messen. Heines Gedicht steht derweil so abseits von Schuberts und Wolfs Gesängen wie ein berühmter Roman abseits von den tapferen Händen der begabtesten Regisseure. 


\title{
Heine, Schubert in Wolf: "Ich stand in dunkeln Träumen"
}

\begin{abstract}
Povzetek
$V$ članku se avtor ukvarja $z$ dvema uglasbitvama Heinejeve pesmi "Ich stand in dunkeln Träumen". Prva je Scbubertova iz leta 1826, druga pa samospev, ki ga je Wolf napisal na isto besedilo maja 1878. Z uglasbitvijo te pesmi stoji Schubert na začetku, Wolf pa pri koncu prvega najmočnejšega "uglasbitvenega" vala Heinejevih pesmi. Schubertov pozni kompozicijski poskus loči od Wolfovega mladostnega dela pol stoletja, $v$ katerem je nova spreminjajoča se kompozicijskozgodovinska izkušnja pustila sledi tudi na obeb uglasbitvab. Ne glede na kompozicijsko-tebnične spremembe tega časa pa vendar ne smemo spregledati enovitosti, $k i$ zvrstno opredeljuje klavirski samospev.

Schubert poudarja recitativno branje besedila in strukturo njegove kitične gradnje, uporablja neznatna glasbena sredstva in tako postavlja $v$ ospredje pesnika. Wolfov išče poudarek drugje. Od pesmi zabteva, da zgodbo sama pripoveduje z glasbenimi sredstvi in se bolj kot Schubert oddaljuje od pesnitve, da bi bolje orisal njeno vsebino.

Wolfove verzije si brez Schubertove ne moremo misliti, saj mladi skladatelj ni imel pred očmi le Heinejevega predloga, temveč tudi Schubertovo skladbo, ki mu je bila tako breme kot kot izziv.
\end{abstract}

